\title{
USO FORRAJERO DEL FOLLAJE DE MANDIOCA: UN CAMBIO DE PARADIGMA PRODUCTIVO PARA UN CULTIVO TRADICIONAL DEL NEA
}

\section{Forage use of cassava foliage: a change of productive paradigm for a traditional crop in NEA region}

\author{
Porta, Miriam ${ }^{1}$; Burgos Angela M. ${ }^{2}$; Castelán, María E. ${ }^{1}$; Hack, Claudina M. ${ }^{1}$ \\ ${ }^{1}$ Instituto Agrotécnico Pedro M. Fuentes Godo. FCA - UNNE. Las Heras 727. Resistencia - Chaco. \\ ${ }^{2}$ Cátedra de Cultivos III. FCA - UNNE \\ e-mail: miritaporta@yahoo.com.ar
}

\begin{abstract}
RESUMEN
Los agricultores familiares de Corrientes, han manifestado la necesidad de incorporar nuevas alternativas para la alimentación de su ganado. Los docentes del Instituto Agrotécnico "P. M. Fuentes Godo" y de la Cátedra de Cultivos III de la Facultad de Ciencias Agrarias (UNNE), realizan proyectos de extensión conjunta. Dichos proyectos, enmarcados en el programa "La Universidad en el Medio" y acompañados de tareas de investigación que les dan sustento, fueron iniciados en 2017. Como contraparte se trabajó junto a los asesores técnicos de la Secretaría de Agricultura Familiar. Dos cultivos regionales, mandioca y caña de azúcar, fueron seleccionados para su uso forrajero como pilares del proyecto. Ambos se caracterizan por su bajo requerimiento nutricional, plasticidad ambiental y flexibilidad en los tiempos de cosecha. Las hojas de la mandioca que habitualmente son desechadas en el campo, fueron rescatadas como recurso forrajero revalorizándolas por su aporte proteico. Las tecnologías transferidas en los proyectos han sido: i) Microsilaje mixto de caña de azúcar y hojas de mandioca, que aporta energía y proteínas ii) Plantación de mandioca en alta densidad con fines forrajeros. Las experiencias fueron validadas y las tecnologías en diferentes niveles han sido transferidas y están siendo adoptadas por los productores de la región NEA (Noreste Argentino). Las alternativas propuestas contribuyen a mejorar la actividad ganadera de pequeña escala, permitiendo utilizar y conservar el material vegetal para las épocas de déficit. Los resultados obtenidos han motivado el interés en utilizar particularmente el cultivo de mandioca con un nuevo sistema productivo para un destino forrajero.
\end{abstract}

Palabras Clave: caña de azúcar, mandioca, cultivos for rajeros, microsilos.

\begin{abstract}
In Corrientes, family farmers have expressed the need to incorporate new alternatives for cattle feeding. Teachers from "P. M. Fuentes Godo" Agrotechnical Institute and The Chair of Farming III from the Faculty of Agricultural Sciences at UNNE carry out extension projects together. These projects as part of "La Universidad en el Medio" Program are accompanied by research works that have supported them since 2017. On the other hand, Technical Advisors from the Ministry of Family Farming have worked together as well. Two regional crops, cassava and sugar cane, were selected for forage use as structural axes of the project. Both crops are characterized by having low nutritional requirements, environmental plasticity and flexibility in harvest times. Cassava leaves, which are usually discarded in the field, were retrieved as forage resources revalidated by their protein content. The technologies transferred in the projects have been: i) Mixed microsilage of sugarcane and cassava leaves which provide energy and protein ii) High density cassava plantation for forage purposes. The experiences were validated, technologies have been transferred and are being adopted by regional farmers from NEA (NorthEast Argentina). The proposed alternatives contribute to small-scale livestock activity improvement, allowing forage to be used and preserved during shortage times. The results obtained have motivated the interest in particularly using cassava farming with a new production system for forage destination.
\end{abstract}

Key Words: sugar cane, cassava, for age crops, microsilages.

Recibido: 23/abr/2020. Aceptado: 29/jun/2020 


\section{INTRODUCCIÓN}

Las condiciones agroecológicas de la región del nordeste argentino (NEA), resultan ideales para algunos cultivos rústicos y nutritivos que pueden utilizarse para la producción ganadera como la caña de azúcar (Saccharum officinarum L.) y la mandioca (Manihot esculenta Crantz). Analizando la situación de la agricultura familiar en la provincia de Corrientes, se estima que los pequeños productores representan el $72 \%$ de los productores totales. En sus sistemas productivos, los cultivos que generalmente componen la "sementera baja", fundamentales en la dieta de la familia, están representados por: mandioca, batata, cebolla y maíz.

En la provincia de Corrientes existen 705,6 ha implantadas con mandioca, cuya producción se destina en su mayor parte al autoconsumo y el excedente se comercializa en los pueblos cercanos y ferias francas (CFI, 2019). Por su parte, la actividad ganadera tiene gran trascendencia para la Provincia de Corrientes dado que ocupa el 93\% del total de la superficie agropecuaria (Acosta et al., 2009). Actualmente la estructura de la unidad doméstica familiar hizo que en muchos casos la principal estrategia de obtención de ingresos sea la cría (terneros para recría en campos de mayor superficie) y venta de ganado vacuno (Ramilo y Prividera, 2013). Las pequeñas unidades productivas familiares utilizan los animales como fuerza de trabajo, para autoconsumo y también como fuente de ingresos a través de la venta. Es por ello que los animales ocupan un lugar fundamental en la estructura productiva.

Sin embargo, el principal inconveniente para la ganadería del NEA, es que la producción de forraje durante el año no es constante y está condicionada por factores ambientales estacionales. En primavera y verano el recurso forrajero es abundante y de buen valor nutritivo, pero durante la época invernal es de menor calidad y escasa cantidad.

Una necesidad de la ganadería actual es buscar fuentes de alimento más económicas para formular la dieta de los animales, además de implementar sistemas prácticos de alimentación animal. Esto involucra la producción de materias primas que cumplan ciertos requisitos para los productores como son: elevadas producciones por hectárea, adaptabilidad al medio y fuente de nutrientes (Gil Llanos, 2015).

Una alternativa para superar el déficit invernal de forrajes es la confección de reservas con productos obtenidos en momentos de mayor disponibilidad. Los materiales que podrían ensilarse en este contexto para un suministro diferido en la época de escasez de forrajes frescos (Uset, 2009), serían: a) caña de azúcar y b) hojas de mandioca. La caña de azúcar es un recurso importante por su alta producción de materia seca y su contenido de energía. Las hojas de mandioca, que generalmente se desperdician en el campo, pueden utilizarse para alimentar el ganado por su valiosa concentración de proteína bruta (PB) que puede alcanzar en promedio un $20 \%$ (Scott, 1991; Soares et al., 2016).

Los conocimientos adquiridos y desarrollados entre la Cátedra de Cultivos III y el Instituto Agrotécnico "Pedro M. Fuentes Godo" de la Facultad de Ciencias Agrarias UNNE, sobre calidad nutricional de hojas de mandioca y confección de microsilos mixtos a base de caña de azúcar y mandioca, indican que estos recursos pueden ofrecer forraje conservado en el tiempo, nutricionalmente valioso, basado en tecnologías sencillas y de muy bajo costo. Otro de los desafíos propuesto a los productores y sin duda de mayor innovación, ha sido cambiar el paradigma de la producción del cultivo de mandioca, implantándola exclusivamente para la cosecha de hojas de altísimo valor nutricional con fines forrajeros, donde las raíces reservantes se vuelven el subproducto del cultivo. En este planteo se cosechan en cortes sucesivos las hojas del tercio superior de las plantas, que pueden ser suministradas en fresco con previo oreado, ensiladas o henificadas (Gil Llanos, 2015).

Dado que la transferencia de conocimientos y tecnologías se realiza para satisfacer una demanda específica, la participación activa y conjunta de los demandantes y los técnicos, resulta en la factibilidad de su adopción y éxito. El presente trabajo relata las experiencias de transferencia en la elaboración de microsilos y plantación de mandioca con destino forrajero que los técnicos del Instituto Agrotécnico Fuentes Godo y docentes y alumnos de la Cátedra de Cultivos III de la FCA de la UNNE han realizado en el período 2017-2019.

\section{OBJETIVOS}

- Fomentar en los pequeños productores el uso de las hojas de mandioca consideradas un subproducto del cultivo de raíces, como fuente proteica para la alimentación animal. 
Porta, M. et al.: Uso forrajero del follaje de mandioca.

- Difundir la técnica de confección de microsilos bolsa de hojas de mandioca y caña de azúcar como reserva forrajera.

- Capacitar a productores en el manejo de los cultivos de mandioca con destino forrajero.

- Generar espacios de aprendizaje y capacitación participativa entre profesores, técnicos, investigadores, productores y estudiantes universitarios de grado y posgrado de la FCA y FCV de la UNNE y como también preuniversitarios de escuelas técnicas.

\section{METODOLOGÍA}

Desde 2017 se trabaja a través de proyectos del programa "La Universidad en el Medio" de la UNNE, que se desarrollaron en la Provincia de Corrientes: "Reservas forrajeras de Caña de Azúcar y Mandioca en agroecosistemas de Agricultura familiar" (Resolución 290/18 C.S.) a demanda de Asociación Agricultores Familiares del Departamento San Cosme y "Sistemas de producción y conservación de Caña de azúcar y follaje de batata y mandioca como alternativas para la suplementación ganadera en el NEA" (Resolución 194/19 C.S.), a demanda de la Unión de Productores del Departamento de San Luis del Palmar (UNIPRO) y la Cooperativa Apícola y de Granjas Santa Ana, respectivamente. En ambos casos, se trabajó en conjunto con la Secretaría de Agricultura Familiar de Nación, apoyados por el Ministerio de Producción de la Prov. de Corrientes a través del Acuerdo de Trabajo (Resolución 9442/16 y 10772/19 C.D.).

La propuesta metodológica general planteada fue generar espacios de trabajo interdisciplinario entre ingenieros agrónomos y médicos veterinarios, estudiantes de ingeniería agronómica, técnicos y productores de la Provincia de Corrientes para llevar adelante prácticas del cultivo de mandioca con destino forrajero y confección de reservas (microsilos).

Fue realizado un estudio de diagnóstico previo, para analizar las posibilidades reales de manejo y adopción de tecnologías a la medida de las posibilidades de los productores demandantes. Se propusieron las siguientes tecnologías de fácil adopción por parte de los productores:

A- Elaboración de microsilos con hojas de mandioca y caña de azúcar.

B- Cultivo de mandioca forrajera con alta densidad de plantación.

Se establecieron unidades demostrativas (UD) de mandioca y caña de azúcar en las instalaciones del Campo Didáctico Experimental de la FCA UNNE, ubicado la vera de la Ruta Nacional № 12, Km 1031 en el Dpto. Capital de la Prov. de Corrientes.

A- Elaboración de microsilos de mandioca y caña de azúcar.

Para su realización se tuvo en cuenta la utilización de materiales de bajo costo y fáciles de conseguir por los productores. Como ser bolsas de plastillera, bolsas de consorcio, picadora electrostática. El material cosechado fue molido y mezclado en distintas proporciones (p:p) mandioca: Caña $(30: 70,50: 50,70: 30)$ y hojas de mandioca pura. El material se compactó por capas hasta completar la bolsa de plastillera, que luego se cerró y se cubrió con bolsas de consorcio. Se procedió a extraer todo el aire por medio de una aspiradora manual para lograr la mayor hermeticidad. Una vez confeccionados los microsilos se llevaron a un galpón donde se almacenaron por 60 días, momento a partir del cual ya estaban en condiciones de ser suministrados a los animales.

B- Cultivo de mandioca forrajera con alta densidad de plantación.

Otra técnica propuesta es la utilización de mandioca con fines netamente forrajeros, modificando el sistema de cultivo (marco de plantación a 50 x $50 \mathrm{~cm}$ ). La elección de esta alternativa se basa principalmente en el alto valor nutritivo de las plantas de mandioca, su alto potencial productivo, y a su adaptación a las condiciones edáficas y climáticas de la zona. Se instalaron unidades demostrativas en la FCA UNNE, donde se midió rendimiento de biomasa aérea y su calidad forrajera, mediante cortes del tercio superior cada 60 días. Esta tecnología ve a las hojas de mandioca como producto principal, siendo las raíces un subproducto que se cosecha a los 2 años. En este caso, las raíces también son utilizadas para alimentación animal o bien para industria.

\section{RESULTADOS}

Los resultados de los análisis químicos de laboratorio obtenidos de los silos a los 60 días de confeccionados se presentan en la Tabla 1: 
Tabla 1. Caracterización Nutricional de Microsilos bolsas mixtos en diferentes proporciones porcentuales (\%) de hojas de mandioca (M) con caña de azúcar (C) y de mandiocas puros referidos a \% de Materia Seca (MS), Fósforo (P), Potasio (K), Calcio (Ca), Magnesio (Mg), Fibra detergente neutra (FDN), Fibra detergente ácida (FDA) y Proteína Bruta (PB).

\begin{tabular}{cccccccccc}
\hline Tratamiento & $\mathbf{p H}$ & $\mathbf{\% M S}$ & $\mathbf{\% P}$ & $\mathbf{\% K}$ & $\mathbf{\% C a}$ & $\mathbf{\% M g}$ & \%FDA & \%FDN & \%PB \\
\hline $30 \% \mathrm{M}+70 \% \mathrm{C}$ & 3,56 & 21,60 & 0,15 & 0,99 & 1,96 & 0,29 & 48,61 & 76,56 & 10,20 \\
$50 \% \mathrm{M}+50 \% \mathrm{C}$ & 3,57 & 22,10 & 0,19 & 1,11 & 1,96 & 0,27 & 47,25 & 67,95 & 13,73 \\
$70 \% \mathrm{M}+30 \% \mathrm{C}$ & 3,67 & 24,40 & 0,20 & 1,13 & 2,02 & 0,42 & 39,70 & 61,26 & 15,69 \\
$100 \%$ Mandioca & 3,93 & 18,50 & 0,40 & 1,50 & 2,32 & 0,27 & 34,50 & 62,02 & 18,23 \\
\hline
\end{tabular}

Nuestros resultados coinciden con los trabajos publicados por Howeler (2014) y Scott (1992) donde indican que en los cultivos forrajeros de mandioca encontraron porcentajes de proteína bruta en hojas jóvenes del orden de $20 \%$ y valores de hasta $11 \%$ en hojas maduras de cultivos para cosecha de raíces. Por lo tanto, las hojas de mandioca que eran desperdiciadas por los productores, fueron revalorizadas como subproducto del cultivo por su aporte proteico para la dieta de los animales.

Los resultados obtenidos, refuerzan aún más los trabajos llevados a cabo por Uset (2009) en el INTA de Misiones con silos de cultivos de caña y mandioca y por Fernández Mayer (2014) quien cita que el ensilaje de hojas de mandioca es el procedimiento más sencillo y eficaz, que no solo reduce la concentración de $\mathrm{HCN}$ a valores sin riesgo para los animales monogástricos, sino también permite conservar el valor nutritivo de las hojas para ser usadas en épocas críticas.

A través de los proyectos de extensión se formalizó el trabajo en equipo interdisciplinario y en la bajada a terreno con los diversos actores de la producción. Se logró que los productores "descubran", conozcan y valoren el silaje del follaje de mandioca en combinación con la caña de azúcar. Con ello no solo se han acercado nuevas tecnologías a los agricultores familiares, adaptando la técnica del ensilaje, sino que además se favoreció la incorporación de nuevos conocimientos a las fórmulas más ancestrales de cultivo. El beneficio de realizar los microsilos bolsas no es solo por ser una alternativa para las épocas de déficit sino también porque son factibles de implementar, de muy bajo costo y permiten suministrar las raciones diarias que son de fácil acarreo a los comederos. A partir de las capacitaciones y a la difusión mediada por diversos técnicos participantes, los productores de la zona adoptaron esta tecnología en sus establecimientos.

Por otro lado, el cultivo de mandioca plantado en alta densidad (40.000 plantas $\left.\mathrm{ha}^{-1}\right)$ con destino forrajero, con cosechas realizadas cada 60 días propuestas por Gil Llanos (2015) se pudo utilizar exitosamente para la confección de silos, obteniendo materia prima con $20 \%$ de PB (Tabla 2).

Tabla 2. Caracterización nutricional de hojas de mandioca del tercio superior cosechadas cada 60 días y plantadas en alta densidad $\left(40.000 \mathrm{pl} \mathrm{ha}^{-1}\right)$ en Corrientes.

\begin{tabular}{ccccccccc}
\hline Tratamiento & $\% \mathrm{MS}$ & $\% \mathrm{P}$ & $\mathbf{\% K}$ & $\% \mathrm{Ca}$ & $\mathbf{\% M g}$ & $\% \mathrm{FDA}$ & $\mathbf{\% F D N}$ & $\% \mathrm{~PB}$ \\
M. Forrajera & 23 & 0,42 & 1,53 & 2,28 & 0,4 & 49,82 & 62,73 & 22,53 \\
\hline
\end{tabular}

\%Materia Seca (MS), Fósforo (P), Potasio (K), Calcio (Ca), Magnesio (Mg), Fibra detergente neutra (FDN), Fibra detergente acida (FDA) y Proteína Bruta (PB).

Una evaluación del valor nutritivo de cuatro variedades de mandioca en Brasil, sugiere contenidos de proteína bruta (base seca) en planta entera (parte aérea), que varían entre 9,8 y 13,4 \%; mientras en la fracción correspondiente al tercio superior de la planta, este valor osciló entre 15,4 y $18,8 \%$. Valores promedio de diferentes fracciones de la parte aérea de la planta sugieren contenidos de FDN entre 63,3 y 66,9\% y de FDA entre 42,5 y 45,7\% (Souza et al., 2011). Si bien los valores de proteína del tercio superior de la planta que se obtuvieron son superiores (Tabla 2) tanto el porcentaje de FDN y FDA son similares a los mencionados por Souza et al. (2011). De todas maneras, con un contenido superior a 18\% de proteína bruta podríamos decir que el tercio 
Porta, M. et al.: Uso forrajero del follaje de mandioca.

superior de la planta de mandioca estaría entre los forrajes denominados fibrosos-proteicos. Estos resultados son altamente positivos ya que amplían las posibilidades de mejorar la alimentación animal en predios de agricultura familiar.

El desarrollo de este proyecto promovió un cambio de paradigma en las técnicas de producción y en el uso y tratamiento de productos y subproductos de mandioca y caña, revalorizándolos para fines forrajeros en términos cuanti-cualitativos.

Con los resultados de los proyectos se realizaron jornadas de capacitaciones dirigidas a productores, técnicos, alumnos universitarios y de escuelas Agrotécnicas de la región. En ellas se realizaron demostraciones dinámicas de maquinaria a campo con picadora y embutidora de forraje, su regulación y análisis de potencial.

Las jornadas actuaron como espacios de intercambio, retroalimentación de conocimientos, saberes y de motivación para nuevos productores que se acercaban por curiosidad.

Se logró la apropiación de la tecnología de confección de microsilos bolsa usando caña de azúcar y hojas de mandioca en distintas proporciones.

Se generó un valioso espacio de aprendizaje interdisciplinar que ha permitido presentar trabajos en congresos y reuniones de extensión y que se ha sustanciado en la formación de recursos humanos de grado y posgrado.

\section{BIBLIOGRAFÍA}

Acosta, F., Giménez, L., Richieri, C. y Calvi, M. (2009). Zonas Agroeconómicas Homogéneas. Corrientes. INTA. Estudios socioeconómicos de la sustentabilidad de los sistemas de producción y recursos naturales $\mathrm{N}^{\circ} 8: 75 \mathrm{pp}$.

CFI. (2019). Proyecto: "Recuperación y desarrollo productivo de los cultivos de batata y mandioca de la provincia de Corrientes"- 168 págs.

Fernández Mayer, A. (2014). Transformación de subproductos y residuos de agroindustria de cultivos templados, subtropicales y tropicales en carne y leche bovina. Ediciones INTA. Boletín Técnico N²0: 200 pp.

Gil Llanos, J.L. (2015). Uso de Yuca en la alimentación animal. Corporación CLAYUCA. Palmira, Colombia. Cartilla Módulo 3. 16 pp. Recuperado de: http://www.clayuca.org/sitio/images/publicaciones/ cartilla modulo 3 yuca alimentacion animal.pdf

Howeler, R. (2014). Sustainable soil and crop management of cassava in Asia: a refer ence manual. CIAT Publication $N^{\circ}$ 389. Cali, CO: Centro Internacional de Agricultura Tropical (CIAT). 280 págs.

Ramilo, D.N. y Prividera, G. (2013). La agricultura familiar en la Argentina: diferentes abor dajes para su estudio. Ediciones INTA. Estudios socioeconómicos de los Sistemas Agroalimentarios y Agroindustriales: 316 págs.

Scott, G.J. (1991). Sweet potatoes as animal feed in developing countries: present pattern and future prospects. In: Machin, D. and Nyvold, SEEds. Roots, Tubers, Plantains and Bananas in Animal Feeding. FAO Animal Production and Health Papers 95: 183- 202.

Soares, I.A., Téo, M.S., Debastiani, C., Retuci,V.S. y Baroni, S. (2016). Concentrado proteico obtido das folhas de Mandioca (Manihot esculenta Crantz) de tres variedades comerciales. Acta Ambiental Catarinense. Vol. 13 (1): 1-7. DOI: http://dx.doi.org/10.24021/raac.v13i1.3644

Souza A.S., Rocha Jn. V.R., Mota A.D., Palma M.N., Franco M.O., Dutra E.S. Santos C.C., Aguiar A.C., Oliveira C.R. y Rocha E.J. (2011). Valor nutricional de frações da parte aérea de quatro variedades de mandioca. Rev. Bras. Saúde Prod. An. 12 (2): 441-455.

Uset, O.A. (2009). Utilización de raíces y parte aérea de mandioca en la alimentación animal. EEA NTA Montecarlo (Misiones, Argentina). Informe Técnico 62: 6-11. 